\title{
LiTRE-UFF: UMA EXPERIÊNCIA DE ENSINO, PESQUISA E EXTENSÃO NO TRAUMA
}

\author{
Henrique Figueiredo Ottoni ${ }^{1}$ \\ Filipe Moreira de Andrade ${ }^{2}$
}

\begin{abstract}
RESUMO: Ligas acadêmicas são organizações estudantis que promovem atividades didáticas, científicas, culturais e sociais para seus membros. A difusão e a atualização do conhecimento sobre o trauma, com treinamento teórico e prático sobre as emergências médicas mais comuns, são medidas de grande impacto para a melhoria da qualidade e eficácia da assistência à vítima, sendo esse o papel das ligas de trauma. A Liga de Trauma, Reanimação e Emergência da Universidade Federal Fluminense (LiTRE-UFF) iniciou suas atividades em 2008 e, desde então, organiza-se em três áreas de atuação: prevenção e atuação comunitária, pré-hospitalar e intra-hospitalar. Com seis anos de experiência em ensino, pesquisa e extensão, a LiTRE já organizou cursos e atividades de pesquisa e extensão, ofereceu 360 aulas teóricas e 90 práticas a seus ligantes, além de 45 apresentações de trabalhos em congressos. A atuação da LiTRE junto aos estudantes de Medicina e Enfermagem da UFF possibilita formação técnica e humanística diferenciada e, atuando junto à sociedade e aos profissionais de saúde, promove melhoria da qualidade da assistência às vítimas de trauma e emergências médicas.
\end{abstract}

PALAVRAS-CHAVE: Medicina de emergência. Ligas acadêmicas. Educação. Relação comunidade-universidade.

\section{LiTRE-UFF: an experience on teaching, research and extension on trauma}

\begin{abstract}
Academic Leagues are student organizations that promote scientific, cultural, social and learning activities for their members. Spreading and updating Trauma-related knowledge, with theoretical and practical training regarding the most common medical emergencies, are measures of great impact on the improvement of the quality and efficiency of the assistance provided to victims - and this is the role played by Trauma Leagues. The Trauma, Reanimation and Emergency League of the Fluminense Federal University (LiTREUFF) began its activities in 2008, which have been acted in 3 fields: Prevention and Community Service, Pre-Hospital, and In-Hospital. With 6 years of teaching experience, LiTRE has organized courses and research activities and assistance, 360 theoretical classes and 90 hands-on sessions to its connections, as well as 45 papers presented in conferences. LiTRE's actions alongside Medical Sciences and Nursing students of the Fluminense Federal University enables a distinct technical and humanistic training which, together with medical professionals and society, promotes an improvement in the quality of assistance provided to victims of trauma and medical emergencies.
\end{abstract}

KEYWORDS: Emergency medicine. Ligas acadêmicas. Education. Community-university relations.

\footnotetext{
${ }^{1}$ Graduado em Medicina pela Universidade Federal Fluminense (henriqueottoni@hotmail.com).

${ }^{2}$ Doutor em Ciências Médicas pela Universidade Federal Fluminense, professor de Cirurgia Torácica no Departamento de Medicina e Enfermagem da Universidade Federal de Viçosa (filipetorax@hotmail.com). 


\section{INTRODUÇÃO}

As ligas acadêmicas são associações formadas e geridas por estudantes, nas quais se busca promover o conhecimento em áreas específicas não contempladas na grade curricular tradicional ou, ainda, aprofundar a discussão de matérias de interesse já abordadas durante o curso de graduação (NASR et al., 2012). Para tanto, as ligas acadêmicas se orientam segundo o princípio da indissociabilidade entre ensino, pesquisa e extensão (TORRES et al., 2008) e contam com a supervisão de profissionais e professores vinculados a uma Instituição de Ensino Superior (IES) ou, no caso das ligas das Ciências da Saúde, vinculados a um hospital de ensino (MONTEIRO et al., 2008).

No Brasil, as ligas estão presentes, principalmente, na área da Medicina, mas existem grupos universitários atuando em outras áreas da saúde, como Enfermagem, Nutrição e Fonoaudiologia, assim como nas Ciências Humanas e Exatas. Por aqui, esse modelo de associação universitária surgiu em 1920, com a criação da Liga de Combate à Sífilis da Faculdade de Medicina da Universidade de São Paulo. Nessa época, os estudantes passaram a se reunir em grupos de estudos com a finalidade de aprofundar os conhecimentos sobre a sífilis e começaram a atuar com a comunidade externa à universidade, montando postos de profilaxia e tratamento gratuito à população (USP, 2014).

Nos últimos anos, houve um aumento considerável do número de ligas acadêmicas por todo o país (PÊGO-FERNANDES et al., 2011; FILHO et al., 2010). Esse fenômeno motivou a criação da Associação Brasileira de Ligas Acadêmicas de Medicina, vinculada à Associação Brasileira de Educação Médica, no ano de 2005, tendo como objetivos integrar e estimular a troca de experiências entre as já existentes, estimular a criação de novas e conferir um padrão de qualidade entre as associadas (NASR et al., 2012).

Atualmente, no Brasil, o trauma é a maior causa de morte nas primeiras quatro décadas de vida, acarretando custos sociais e financeiros elevados (GAWRYSZEWSKI, 2004). A educação continuada de estudantes e profissionais de saúde é essencial para que o manejo do trauma e sua prevenção sejam realizados de maneira mais eficiente. A atualização continuada é fundamental, uma vez que os protocolos de manejo são frequentemente reformulados a partir do conhecimento gerado com os novos estudos e publicações sobre o tema. A educação da população, efetuada por meio da extensão universitária, objetiva à diminuição das vítimas de trauma, bem como à maior efetividade em seu atendimento inicial.

Em 1992, foi criada, na Universidade Estadual de Campinas, pelos médicos Mario Mantovani e Admar Concon Filho, a primeira Liga de Trauma registrada no Brasil (UNICAMP, 2014). Concomitantemente, houve a criação de inúmeras outras em todo o país e, no ano de 2003, surgiu o Comitê Brasileiro das Ligas do Trauma (CoBraLT), uma instituição estudantil com a finalidade de unir e incentivar as ligas do trauma das faculdades de Medicina, Enfermagem e demais áreas da saúde do Brasil (COBRALT, 2014). Atualmente, estima-se a existência de 150 delas no país, com o objetivo comum de aprofundar os estudos sobre o trauma, gerar pesquisa científica sobre o tema e atuar junto à comunidade na prevenção de novos eventos e na difusão do conhecimento. 


\section{OBJETIVOS}

Realizamos uma avaliação da experiência de seis anos de atividades de acadêmicos de Medicina e Enfermagem organizados na Liga Acadêmica de Trauma, Reanimação e Emergência (LiTRE) no Hospital Universitário Antônio Pedro (HUAP), pertencente à Universidade Federal Fluminense (UFF), sediada em Niterói-RJ, Brasil.

\section{METODOLOGIA}

Os dados referentes à experiência e atuação da LiTRE foram obtidos a partir dos registros de reuniões e atividades promovidos pela Liga, que se encontram disponíveis sob forma de atas.

\section{Relato da experiência}

O HUAP foi inaugurado no dia 15 de janeiro de 1951 e denominado Hospital Municipal Antônio Pedro. Durante os seus primeiros anos de existência, foi administrado e financiado pela Prefeitura de Niterói, mantendo-se assim até 1964, quando, após uma longa mobilização dos estudantes de Medicina da UFF, a unidade foi cedida pela prefeitura à UFF, tornando-se, assim, Hospital Universitário Antônio Pedro.

A história do serviço de emergência do HUAP tem seu marco histórico no final de 1961. Após passar cinco anos fechado por escassez de recursos, o hospital foi reaberto, em dezembro de 1961, em caráter de emergência, para atender às vítimas do incêndio do Gran Circus Americano, que vitimou 400 pessoas, a maioria delas crianças. Uma equipe composta por médicos da prefeitura e estudantes da Faculdade de Medicina da UFF foi montada, emergencialmente, para viabilizar esse atendimento. Esse episódio foi decisivo para a criação do Serviço de Emergência do HUAP. Dessa maneira, a LiTRE adota como marco simbólico de sua fundação a data 17 de dezembro de 1961, dia do incêndio do Gran Circus Americano, como forma de homenagear os estudantes e profissionais que atuaram naquele episódio e iniciaram a trajetória da UFF e do HUAP em relação ao atendimento de urgência e emergência.

Atualmente, o HUAP é a maior e mais complexa unidade de saúde da Grande Niterói e, portanto, considerado na hierarquia do SUS como hospital de nível terciário e quaternário. $\mathrm{O}$ serviço de emergência atende à população da Zona Metropolitana II, que engloba, além de Niterói, as cidades de Itaboraí, Maricá, Rio Bonito, São Gonçalo, Silva Jardim e Tanguá. Sua área de abrangência atinge uma população estimada em mais de dois milhões de habitantes.

Nesse contexto, é fundada, em 2008, a LiTRE-UFF, com a missão de estimular o estudo das emergência médicas e do trauma em toda comunidade acadêmica da UFF, visando melhor formação dos alunos e qualidade no atendimento à sociedade. A LiTRE-UFF é formada e gerida pelos alunos de graduação em Medicina e Enfermagem e é supervisionada por um corpo docente vinculado à universidade e ao HUAP, tendo como coordenador geral o 
professor José Carlos Trugilho, que, atualmente, também ocupa o cargo de diretor da Faculdade de Medicina da UFF.

A LiTRE se organiza em três áreas de atuação: prevenção e atuação comunitária; préhospitalar; e intra-hospitalar. Cada uma delas é formada por um grupo determinado de estudantes de graduação e tem um médico e um professor orientador responsável pela supervisão de todas as atividades.

A área de prevenção e atuação comunitária tem como coordenador o professor Armando Cypriano Pires e objetiva promover a educação continuada de comunidades e profissionais de saúde; formar socorristas leigos; promover atividades didáticas e de campo; desenvolver trabalhos científicos e discussão de artigos. Atualmente, fazem parte dessa área alunos de gradução de Medicina e Enfermagem entre o $3^{\circ}$ e o $5^{\circ}$ períodos de seus cursos, selecionados por meio de um processo seletivo realizado anualmente.

Ao longo desses seis anos, a área de prevenção e atuação comunitária realizou diversas atividades (Tabela 1), como o apoio a outras ligas acadêmicas de trauma do Rio de Janeiro na organização de oficinas de ensino de primeiros socorros. Houve, ainda, organização de campanhas de doação de sangue ao banco de sangue do HUAP e parceria com a Companhia Niterói Transporte e Trânsito (NitTrans) e com a Concessionária CCR Via Lagos (concessionária que administra a rodovia RJ-124, que conecta os municípios de Rio Bonito a São Pedro da Aldeia) em campanhas comunitárias de prevenção de acidentes de trânsito na Semana Nacional do Trânsito.

A Liga promoveu, ainda na área de prevenção e atuação comunitária, cursos de Suporte Básico de Vida (BLS), para profissionais do HUAP e de postos de saúde de Niterói e do Rio de Janeiro e aos soldados da Força Aérea Brasileira (FAB), e parceria com a Prefeitura Municipal de Niterói e com o Serviço de Atendimento Móvel de Urgência (SAMU - Metropolitana II) no projeto "Samu Mirim". Esse projeto tem como objetivo realizar visitas às escolas públicas do município de Niterói para orientar estudantes de quando e como utilizar o serviço 192, abordar os efeitos dos trotes telefônicos sobre o funcionamento do SAMU, trabalhar temas relativos à prevenção de acidentes de trânsito e BLS e oferecer treinamento sobre como realizar um primeiro atendimento às emergências mais comuns no ambiente residencial e escolar.

Tabela 1 - Atividades realizadas na área de prevenção e atuação comunitária da LiTRE em 2013.

\section{Área de Prevenção e Atuação Comunitária}

\begin{tabular}{lccc}
\hline Atividade & $\begin{array}{c}\text { Número de alunos } \\
\text { envolvidos }\end{array}$ & $\begin{array}{c}\text { Descrição da } \\
\text { atividade }\end{array}$ & Período \\
& & $\begin{array}{c}\text { Elaboração de } \\
\text { material para } \\
\text { divulgação da } \\
\text { campanha e }\end{array}$ & \\
Campanha de & Medicina: 18 & incentivo dos & março de 2013 \\
doação de sangue ao & Enfermagem do 12 & HUAP e & \\
Banco de Sangue do & estudantes da \\
HUAP & UFF à doação \\
& de sangue
\end{tabular}




\begin{tabular}{|c|c|c|c|}
\hline $\begin{array}{l}\text { Ação de prevenção } \\
\text { de acidentes de } \\
\text { trânsito na Semana } \\
\text { Nacional do Trânsito }\end{array}$ & $\begin{array}{l}\text { Medicina: } 12 \\
\text { Enfermagem: } 6\end{array}$ & $\begin{array}{c}\text { Elaboração de } \\
\text { material para } \\
\text { divulgação da } \\
\text { campanha e } \\
\text { três aulas } \\
\text { sobre } \\
\text { prevenção de } \\
\text { acidentes de } \\
\text { trânsito para a } \\
\text { comunidade }\end{array}$ & $\begin{array}{c}19,20 \text { e } 21 \text { de } \\
\text { setembro de } 2013\end{array}$ \\
\hline $\begin{array}{l}\text { Curso de BLS para } \\
\text { profissionais do } \\
\text { HUAP }\end{array}$ & $\begin{array}{l}\text { Medicina: } 15 \\
\text { Enfermagem: } 4\end{array}$ & $\begin{array}{c}\text { Aulas } \\
\text { semanais de } \\
\text { atualização } \\
\text { sobre BLS } \\
\text { para } \\
\text { profissionais } \\
\text { de saúde do } \\
\text { HUAP }\end{array}$ & novembro de 2013 \\
\hline $\begin{array}{l}\text { Curso de BLS para } \\
\text { os soldados da FAB }\end{array}$ & $\begin{array}{c}\text { Medicina: } 8 \\
\text { Enfermagem: } 2\end{array}$ & $\begin{array}{c}\text { Aula de } \\
\text { atualização } \\
\text { sobre BLS } \\
\text { para soldados } \\
\text { da FAB }\end{array}$ & agosto de 2013 \\
\hline Samu Mirim & $\begin{array}{l}\text { Medicina: } 12 \\
\text { Enfermagem: } 6\end{array}$ & $\begin{array}{c}\text { Aulas em } 4 \\
\text { escolas } \\
\text { municipais em } \\
\text { Niterói sobre o } \\
\text { funcionamento } \\
\text { do serviço } \\
\text { 192, efeitos } \\
\text { negativos dos } \\
\text { trotes } \\
\text { telefônicos e } \\
\text { prevenção de } \\
\text { acidentes }\end{array}$ & $\begin{array}{c}\text { março de } 2013 \text { a } \\
\text { junho de } 2013\end{array}$ \\
\hline Aulas & $\begin{array}{c}\text { Medicina: } 20 \\
\text { Enfermagem: } 12\end{array}$ & $\begin{array}{c}\text { Aulas } \\
\text { semanais de } \\
\text { atualização } \\
\text { para os } \\
\text { ligantes }\end{array}$ & $\begin{array}{l}\text { fevereiro de } 2013 \text { a } \\
\text { novembro de } 2013\end{array}$ \\
\hline
\end{tabular}

Fonte: Os autores (2014).

A área de pré-hospitalar tem como coordenador o professor Nisval Magalhães Júnior. Os objetivos são: promover a capacitação dos alunos na área de medicina pré-hospitalar; conscientizar a comunidade acadêmica da importância do atendimento integrado pré e intrahospitalar; atuar em atividades práticas em entidades públicas e privadas; educar a população a valorizar e solicitar de forma correta o serviço pré-hospitalar; e promover atividades didáticas. Atualmente, fazem parte dessa área alunos do $6^{\circ}$ e $7^{\circ}$ períodos de Medicina e alunos cursando entre o $5^{\circ}$ e $7^{\circ}$ períodos de Enfermagem, selecionados por meio de um processo seletivo realizado anualmente. Ao longo desses seis anos, a área realizou diversas 
atividades (Tabela 2), como o Estágio em Ambulância do SAMU Metropolitana II, treinamento de resgate na companhia de atendimento pré-hospitalar rodoviário ENSEG e organização de cursos de extricação.

A área intra-hospitalar tem como coordenador o médico Rodrigo Andrade Vaz de Melo e busca conscientizar a comunidade acadêmica da importância do atendimento integrado pré e intra-hospitalar; permitir estágio supervisionado extracurricular na emergência do HUAP; e promover atividades didáticas acerca dos protocolos atuais em trauma e emergência médica. Atualmente, fazem parte dessa área alunos de graduação de Medicina, cursando entre $07^{\circ} \mathrm{e}$ $9^{\circ}$ períodos, selecionados por meio de um processo seletivo realizado anualmente. Ao longo desses seis anos, a área intra-hospitalar realizou diversas atividades (Tabela 2) como plantões no setor de emergência do HUAP, do Hospital Estadual Azevedo Lima e do Hospital Municipal Lourenço Jorge, além de organização de cursos de sutura aos estudantes de graduação de Medicina da UFF e de cursos sobre protocolos atuais de Trauma, Emergência e Suporte Avançado de Vida em Pediatria (PALS), no Hospital Getúlio Vargas Filho.

Tabela 2 - Atividades realizadas nas áreas de pré-hospitalar e intra-hospitalar da LiTRE em 2013

\section{Área Pré-hospitalar}

\begin{tabular}{|c|c|c|c|}
\hline Atividade & $\begin{array}{l}\text { Número de alunos } \\
\text { envolvidos }\end{array}$ & $\begin{array}{l}\text { Descrição da } \\
\text { atividade }\end{array}$ & Período \\
\hline $\begin{array}{l}\text { Estágio em } \\
\text { Ambulância do } \\
\text { SAMU } \\
\text { Metropolitana II }\end{array}$ & $\begin{array}{l}\text { Medicina: } 10 \\
\text { Enfermagem: } 6\end{array}$ & $\begin{array}{l}\text { Estágio semanal } \\
\text { com duração de } \\
\text { 12h/plantão na } \\
\text { Ambulância do } \\
\text { SAMU } \\
\text { Metropolitana II, } \\
\text { com } \\
\text { acompanhamento } \\
\text { do trabalho dos } \\
\text { profissionais } \\
\text { envolvidos no } \\
\text { setor }\end{array}$ & $\begin{array}{c}\text { fevereiro a } \\
\text { novembro de } 2013\end{array}$ \\
\hline Aulas & $\begin{array}{l}\text { Medicina: } 10 \\
\text { Enfermagem: } 6\end{array}$ & $\begin{array}{l}\text { Aulas semanais } \\
\text { de atualização } \\
\text { para os ligantes }\end{array}$ & $\begin{array}{c}\text { fevereiro a } \\
\text { novembro de } 2013\end{array}$ \\
\hline \multicolumn{4}{|c|}{ Área Intra-hospitalar } \\
\hline Atividade & $\begin{array}{l}\text { Número de alunos } \\
\text { envolvidos }\end{array}$ & $\begin{array}{l}\text { Descrição da } \\
\text { atividade }\end{array}$ & Período \\
\hline $\begin{array}{l}\text { Plantão na } \\
\text { Emergência do } \\
\text { Hospital Municipal } \\
\text { Lourenço Jorge }\end{array}$ & Medicina: 10 & $\begin{array}{c}\text { estágio semanal } \\
\text { com duração de } \\
12 \mathrm{~h} / \text { plantão na } \\
\text { Emergência do } \\
\text { Hospital } \\
\text { Municipal } \\
\text { Lourenço Jorge, } \\
\text { com } \\
\text { acompanhamento } \\
\text { do trabalho dos }\end{array}$ & $\begin{array}{l}\text { fevereiro de } 2013 \\
\text { a julho de } 2013\end{array}$ \\
\hline
\end{tabular}




\begin{tabular}{|c|c|c|c|}
\hline & & $\begin{array}{l}\text { profissionais } \\
\text { envolvidos no } \\
\text { setor }\end{array}$ & \\
\hline $\begin{array}{l}\text { Plantão na } \\
\text { Emergência do } \\
\text { Hospital Estadual } \\
\text { Azevedo Lima }\end{array}$ & Medicina: 10 & $\begin{array}{l}\text { Estágio semanal } \\
\text { com duração de } \\
12 \mathrm{~h} / \text { plantão na } \\
\text { Emergência do } \\
\text { Hospital } \\
\text { Estadual } \\
\text { Azevedo Lima, } \\
\text { com } \\
\text { acompanhamento } \\
\text { do trabalho dos } \\
\text { profissionais } \\
\text { envolvidos no } \\
\text { setor }\end{array}$ & $\begin{array}{l}\text { agosto de } 2013 \text { a } \\
\text { dezembro de } 2013\end{array}$ \\
\hline Aulas & Medicina: 20 & $\begin{array}{l}\text { Aulas semanais } \\
\text { de atualização } \\
\text { para os ligantes }\end{array}$ & $\begin{array}{c}\text { fevereiro de } 2013 \\
\text { a novembro de } \\
2013\end{array}$ \\
\hline
\end{tabular}

Fonte: Os autores (2014).

Algumas atividades não eram específicas de determinada área e podiam contar com a participação de qualquer ligante, como os Simulados de Desastre Aéreo e os Simulados de Desastre com Múltiplas Vítimas com realização do protocolo START (Simple Triage And Rapid Treatment ou Triagem Simples e Tratamento Rápido). Em 2013, realizou-se o curso de salvamento aquático, com aprendizado teórico e prático sobre técnicas de assistência e resgate às vítimas de afogamento. Já na Jornada Mundial da Juventude (JMJ), os ligantes atuaram como socorristas voluntários em postos de atendimento dispostos ao longo das vias de peregrinação, após treinamento da Cruz Vermelha Brasileira (CVB) (Tabela 3).

Tabela 3 - Atividades comuns às três áreas de atuação da LiTRE realizadas em 2013.

\begin{tabular}{|c|c|c|c|}
\hline \multicolumn{4}{|c|}{ Atividades comuns às três áreas de atuação } \\
\hline Atividade & $\begin{array}{l}\text { Número de alunos } \\
\text { envolvidos }\end{array}$ & $\begin{array}{l}\text { Descrição da } \\
\text { atividade }\end{array}$ & Período \\
\hline $\begin{array}{l}\text { Simulados de } \\
\text { desastre aéreo e de } \\
\text { desastre com } \\
\text { múltiplas vítimas }\end{array}$ & $\begin{array}{c}\text { Medicina: } 35 \\
\text { Enfermagem: } 12\end{array}$ & $\begin{array}{c}\text { Curso prático } \\
\text { no aeroporto } \\
\text { do Galeão } \\
\text { sobre } \\
\text { atendimento } \\
\text { de múltiplas } \\
\text { vítimas em } \\
\text { desastre aéreo } \\
\text { com aplicação } \\
\text { do protocolo } \\
\text { START }\end{array}$ & setembro de 2013 \\
\hline $\begin{array}{l}\text { Curso de salvamento } \\
\text { aquático do Dr. }\end{array}$ & $\begin{array}{l}\text { Medicina: } 10 \\
\text { Enfermagem: } 4\end{array}$ & $\begin{array}{l}\text { Curso prático } \\
\text { sobre }\end{array}$ & outubro de 2013 \\
\hline
\end{tabular}


salvamento

aquático

\begin{tabular}{|c|c|c|c|}
\hline $\begin{array}{l}\text { Jornada Mundial da } \\
\text { Juventude (JMJ) }\end{array}$ & $\begin{array}{c}\text { Medicina: } 18 \\
\text { Enfermagem: } 6\end{array}$ & $\begin{array}{c}\text { Atuação dos } \\
\text { ligantes como } \\
\text { socorristas } \\
\text { voluntários em } \\
\text { postos de } \\
\text { atendimento } \\
\text { dispostos ao } \\
\text { longo das vias } \\
\text { de } \\
\text { peregrinação } \\
\text { da JMJ, após } \\
\text { treinamento na } \\
\text { Cruz } \\
\text { Vermelha } \\
\text { Brasileira } \\
\text { (CVB) }\end{array}$ & $\begin{array}{c}23 \text { a } 28 \text { de julho de } \\
2013\end{array}$ \\
\hline LiTRE Saúde & $\begin{array}{l}\text { Medicina: } 20 \\
\text { Enfermagem: } 6\end{array}$ & $\begin{array}{c}\text { Atividade } \\
\text { realizada na } \\
\text { praia de Icaraí } \\
\text { (Niterói-RJ) } \\
\text { para ensinar o } \\
\text { BLS e a } \\
\text { identificação } \\
\text { precoce do } \\
\text { AVC à } \\
\text { população e } \\
\text { esclarecer } \\
\text { sobre a } \\
\text { prevenção do } \\
\text { câncer de pele }\end{array}$ & agosto de 2013 \\
\hline
\end{tabular}

Fonte: Os autores (2014).

Não obstante, a LiTRE apresenta como atividades permanentes, discussões teóricas a cada quinze dias e aulas práticas a cada dois meses, em cada uma das três áreas em que se divide, o que totaliza, aproximadamente, 360 reuniões acadêmicas com aulas temáticas ministradas por médicos ou ligantes e 90 atividades práticas de simulação de eventos de trauma e emergência durante os seis anos de sua existência. As aulas organizadas pela Liga são de participação obrigatória dos ligantes, contando com lista de chamada e controle de frequência. Elas podem ser assistidas por qualquer aluno de graduação da UFF que esteja interessado em aprender e se atualizar sobre trauma e emergências médicas.

Anualmente, com duração de quatro dias, a LiTRE-UFF realiza o seu curso de seleção de ligantes e conta com a exposição de aulas teóricas e práticas sobre diversas emergências médicas, ministradas por médicos experientes e renomados. Ao final do curso, os alunos que desejam ser ligantes realizam uma avaliação teórica de múltipla escolha e uma avaliação prática sobre BLS e, respeitando o número de vagas disponíveis em cada ano, os que obtêm as melhores notas são selecionados e passarão a fazer parte da liga pelo período de um ano. 
Além disso, a LiTRE-UFF organiza, anualmente, o projeto de extensão "LiTRE-Saúde", que conta com o apoio de outras ligas acadêmicas da UFF, como a Liga de Pediatria (LIPE), Liga de Neurologia e Neurociências (LINNEC), Liga de Oncologia Clínica e Cirúrgica (LIONCC) e Liga de Ciências Cardiovasculares (LICCA), direcionado à prevenção de eventos diversos. Durante um final de semana, os alunos da LiTRE e das ligas parceiras se reúnem na Praia de Icaraí, em Niterói-RJ, e, supervisionados por um docente da universidade, atuam na difusão de conhecimentos teóricos e práticos sobre temas como BLS, reconhecimento do Acidente Vascular Encefálico (AVE) e do Infarto Agudo do Miocárdio (IAM), prevenção do câncer de pele e prevenção de acidentes pediátricos. O evento é organizado desde 2011, contando, portanto, com três edições já realizadas.

A Liga mantém, ainda, uma produção científica contínua, que inclui 45 apresentações de trabalhos em congressos regionais, nacionais e internacionais. Participa, desde 2008, do Congresso Brasileiro das Ligas de Trauma (CoLT), organizado pelo CoBraLT, comparecendo, com direito a voto, das assembleias que ocorrem ao final do evento e que definem as estratégias de atuação das ligas afiliadas durante ano seguinte. Em conjunto com as demais ligas de trauma do Rio de Janeiro, a LiTRE-UFF já organizou duas edições do evento, em 2010 e 2012. Outras parcerias entre elas foram a organização do $1^{\circ}$ Congresso Mundial de Trauma, no ano de 2012, realizado na cidade do Rio de Janeiro e, anualmente, a organização do Congresso Pré-CoLT.

A LiTRE é um projeto de extensão institucionalizado pela UFF, contando, desde 2008, com apoio, certificação e bolsa mensal da Pró-reitoria de Extensão da UFF (PROEx-UFF).

\section{CONSIDERAÇÕES FINAIS}

A LiTRE-UFF foi a primeira liga acadêmica criada na UFF, mantendo-se ativa na organização de eventos dentro da universidade, na educação continuada de estudantes e profissionais de saúde e na prestação de serviços à população.

A participação na LiTRE confere ao estudante a possibilidade de obter uma formação diferenciada, na medida em que lhe permite acesso a um estudo teórico e prático aprofundado e atualizado sobre diversos temas dentro do trauma e das emergências médicas. Assim, os futuros médicos e enfermeiros atuarão de forma mais segura e eficiente diante das situações de emergência, contribuindo para a melhoria da assistência aos pacientes e reduzindo a morbimortalidade associada a esses eventos. Por esses motivos, a LiTRE conta, a cada ano, com um número maior de ligantes, na medida em que cresce o interesse de alunos não pertencentes a ela em participar das suas atividades.

Ao longo desses seis anos, a LiTRE-UFF desenvolveu atividades com diversos profissionais, como bombeiros, soldados, agentes de saúde, gestores de saúde, médicos, enfermeiros, técnicos de enfermagem, nutricionistas, dentistas e outros profissionais de saúde das diversas especialidades. Essa experiência permite o reconhecimento da importância do trabalho em equipe multidisciplinar na assistência à vítima de trauma e das emergências médicas. Dessa forma, a participação do estudante na LiTRE possibilita a formação de profissionais éticos, com senso de responsabilidade social e compromisso com a cidadania, capazes de acolher o paciente em sua complexa integralidade biopsicocultural. 


\section{REFERÊNCIAS}

BURJATO JÚNIOR, D. B. História da liga de combate à sífilis e a evolução da sífilis na cidade de São Paulo (1920-1995). 1999. Dissertação (Mestrado) - Faculdade de Medicina da Universidade de São Paulo, São Paulo, 1999.

CoBraLT - Comitê Brasileiro das Ligas do Trauma. Disponível em: <http://www.cobralt .com/>. Acesso em: 14 jan. 2014.

FILHO, P. T. H. et al. Normatização da abertura de ligas acadêmicas: a experiência da Faculdade de Medicina de Botucatu. Revista Brasileira de Educação Médica, Rio de Janeiro, v. 34, n. 1, p. 160-167, jan.-mar. 2010.

GAWRYSZEWSKI, V. P.; KOIZUMI, M. S.; MELLO-JORGE, M. H. P. As causas externas no Brasil no ano 2000: comparando a mortalidade e a morbidade. Cad. Saúde Pública, Rio de Janeiro, v. 20, n. 4, p. 995-1003, jul.-ago. 2004.

MONTEIRO, L. L. F. et al. Ligas acadêmicas: o que há de positivo? Experiência de implantação da liga baiana de cirurgia plástica. Rev. Bras. Cir. Plást., São Paulo, v. 23 n. 3, p. $158-61,2008$.

NASR, A. et al. Prevenção ao trauma e atendimento de qualidade: uma década de liga acadêmica do trauma no hospital do trabalhador - UFPR. Panam J Trauma Critical Care Emerg Surg, Curitiba, v. 1, n. 2, p. 127-130, 2012.

PÊGO-FERNANDES, P. M.; MARIANI, A. W. O ensino médico além da graduação: ligas acadêmicas. Diagn Tratamento, São Paulo, v. 16, n. 2, p. 50-51, 2011.

TORRES, A. R. et al. Academic leagues and medical education: contributions and challenges. Interface - Comunic., Saúde, Educ., Botucatu, v. 12, n. 27, p. 713-720, out.-dez. 2008.

UNICAMP - Universidade Estadual de Campinas. Liga do trauma da UNICAMP. Disponível em: <http://www.insl.com.br/dct/liga.php>. Acesso em: 14 jan. 2014.

USP - Universidade de São Paulo. Faculdade de Medicina. História da liga de combate à sífilis e a outras doenças sexualmente transmissíveis. Disponível em: <http://med.fm.u sp.br/dim/homepage/b105/lcshist.htm>. Acesso em: 14 jan. 2014.

Submetido em 5 de setembro de 2014.

Aprovado em 26 de setembro de 2014. 